\title{
Analysis and Comparison of a Coplanar Waveguide and Micro Strip based Rectenna
}

\author{
S. Banu, A. Vishwapriya, R. Yogamathi, A.V. Meenakshi and \\ S.P.K. Babu
}

\begin{abstract}
In this paper, a modified form of rectenna has been designed for wireless power transmission. Generally, rectenna is nothing but a rectifying antenna which is very much useful for converting RF power into usable DC power. The word 'rectenna' was first introduced by W.C.Brown. Even though there is an enormous application of rectenna, wireless transmission of power is taken into consideration. The necessity of the research lies in the present day trend of power hungry mobile gadgets dying soon and requires frequent charging. Here, the analysis of rectenna with different transmission lines such as micro strip line (MLIN) and coplanar wave guide $(C P W)$ has been made. The performance of rectenna using different transmission lines has been discussed based on factors such as power and voltage. In the analysis it was found that the power conversion efficiency of rectenna based on micro strip line is $65.5 \%$ whereas coplanar waveguide based has $78.5 \%$. Thus rectenna with coplanar waveguide performs well and also more advantageous when compared with MLIN. This new comparative analysis is done in Advanced Design System (ADS) 2009 software from Agilent Technologies. In the design there is no necessity of a low pass filter as the transmission line used is MLIN and CPW. The results are shown as graphs along with the circuit as an ADS realization.
\end{abstract}

Keywords--- Advanced Design System (ADS), Co-Planar Waveguide $(C P W)$, Low Pass Filter (LPF), Monolithic Microwave Integrated Chip (MMIC), Micro Strip line (MLIN), Wireless Power Transmission (WPT)

\section{INTRODUCTION}

$\mathrm{T}$ HE fundamental concept of rectenna is a "rectifying antenna" in which an antenna will be used for receiving RF signal and a rectifying circuit is used to provide DC power[9]-[11]. Technically, it can be treated as a wireless battery, which is a device in need. As the technology is

S. Banu, Student of M.Tech Wireless Communication Systems, Periyar Maniammai University, Thanjavur, India.E-mail: rememberbanu@gmail.com

A. Vishwapriya, Student of M.Tech Wireless Communication Systems, Periyar Maniammai University, Thanjavur, India. E-mail: vishwaa.nivi@gmail.com

R. Yogamathi, Student of M.Tech, Wireless communication systems, Periyar Maniammai University, Thanjavur, India. E-mail: yoga.mathi@yahoo.com

A.V. Meenakshi, Assistant Professor, Department of Electronics \& Communication Engineering, Periyar Maniammai University,Thanjavur, India. E-mail: menu_gow@yahoo.com

S.P.K. Babu, Associate Professor, Department of Electronics \& Communication Engineering, Periyar Maniammai University,Thanjavur,India. E-mail: spkbabu@pmu.edu improving, wireless power plays a vital role in today's life. Now a day's every one prefers the wireless devices such as wireless mouse, wireless battery wireless headphones etc. But we use these types of devices with the help of batteries that would be charged with wired devices. It is obvious that many batteries are being used and a way to charge these batteries wirelessly is desirable. This can be made possible as per the discussions given below.

In earlier system, the rectenna design focuses on four subsystems as shown in fig1: (1) the receiving antenna, (2) the matching sections, (3) the rectification, and (4) the postrectification filter [3]-[16]. The big challenge is how to maximize the power-conversion efficiency of rectenna for input-power levels of, for example, $0 \mathrm{dBm}$ or less. The present need is to produce more dc power than the previous half-wave rectenna and design a weight less rectenna circuit [8].

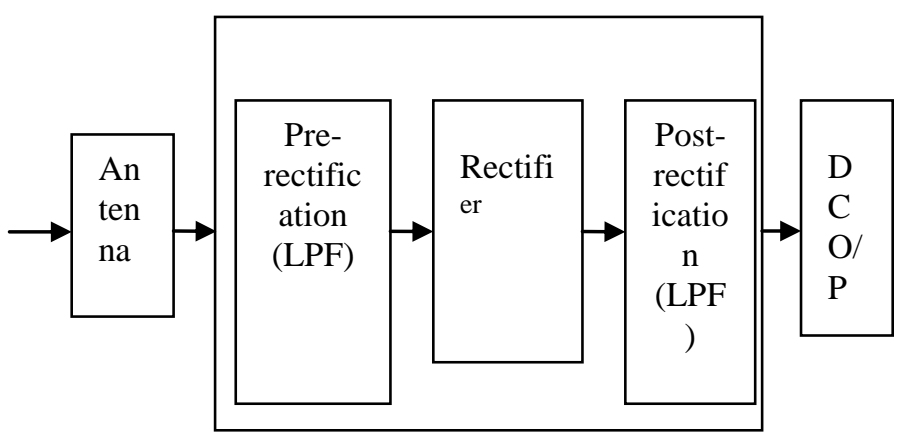

Figure 1: Block Diagram of Rectenna [16]

\section{Pre-Rectification}

From fig 1, which shows the earlier system, it is seen the output of the antenna is directly fed into pre-rectification filter. It allows the operating frequency to flow and prevents interference signals and re-radiation of higher order harmonics which is generated by the diode [11]. It also helps to provide constant load impedance for the antenna.

\section{Rectification}

Rectification plays an important role in the rectenna and so it is called as key function of rectenna (converting microwave rf energy into dc power). The Schottky diode was used as the rectifying device, due to its high switching capability that enables it to follow a high-frequency input signal [11]-[8]. A commercial Avago HSMS8101 was used in the NPS rectenna system. 


\section{Post-Rectification}

A post-rectification filter is used to extract the dc component and produce a smooth transient for output $\mathrm{dc}$ power.

With the above introduction on operation of rectenna, the remaining topics of this research are organized as follows. Section II deals with the rectenna implementation, antenna and usage of schottky diode where as section III says about transmission lines in designing the rectenna. Section IV and V deals about applications of rectenna and simulation results and discussion respectively where as section VI deals about conclusion.

\section{RECTENNA IMPLEMENTATION}

In the earlier days, they designed rectenna with transmission lines and low pass filter [2] (to remove unwanted harmonics and re-radiations of signal) due to the presence of re-radiation in diode and interference in signal. This leads to production of high interference, complexity in design and high cost. The above poor design can be modified by using different transmission lines such as MLIN and CPW which provides high power efficiency. Along with the above modifications, a Schottky diode capable of having high switching frequency removes the re-radiation of signal. So, in this new system, low pass filter is not necessary [7]-[8]. Thus, this system is easier to design, less expensive and has less weight

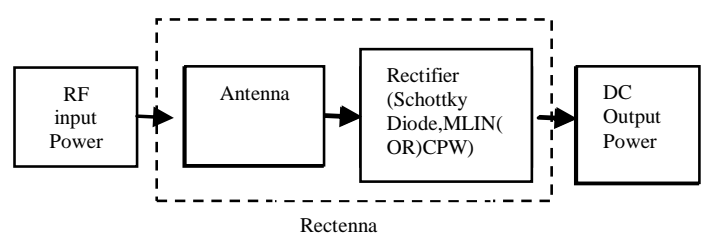

Figure 2: Our Modified Rectenna Implementation

Rectifier is a nonlinear circuit, which converts RF power into DC power [11]. The mathematical relation that describes the conversion efficiency is given by Equation (1) where $V_{D C}$ is dc output voltage, $P_{R F}$ is input power of rectenna and $R_{L}$ is load resistance [16].

$$
\text { efficiency }=\frac{\frac{V_{D C}}{R_{L}}}{P_{R F}} * 100
$$

Antenna design is important in the proposed rectenna since it absorbs incident microwave power, and the rectifier converts it into a useful electric power. In order to reduce the size of the rectenna [11], we eliminate the use of low pass filter and consequently a circular antenna with harmonic rejection was proposed. Equation (2) gives the resonant angular frequency response $\left(\omega_{\mathrm{r}}\right)$ of a circular microstrip patch antenna (CMPA).

$$
\mathrm{J}_{\mathrm{n}}\left(\mathrm{A}_{\mathrm{e}} \cdot \omega_{\mathrm{r}} \sqrt{\varepsilon_{\mathrm{r}} \varepsilon_{0} \mu_{0}}\right)=0
$$

Where $J_{n}(x)$ is the Bessel function of $n$ order, and $A_{e}$ is the effective radius of the patch, $\varepsilon_{\mathrm{r}}$ and $\varepsilon_{0}$ are relative and air permittivity where as $\mu_{0}$ be the vacuum permeability.

\section{Antenna}

Antennas are transducers which are used in wireless communication systems. It consists of many types based on their characteristics such as half-wave dipole antenna, horn antenna, parabolic antenna, and micro strip antenna to name a few. Some of these antennas may not be suitable for the research undertaken due to their physical characteristics such as weight, size, cost etc.

It is found that circular patch antenna [6], due to its capability of reducing the re- radiation of harmonics, is found to be suitable. The response of a circular-patch antenna along with the, the return loss of a circular-patch antenna about 50 $\mathrm{dB}$ where as in practice, the return loss is likely to be $15 \mathrm{~dB}$ to $20 \mathrm{~dB}$ is also given in the literature [9].

\section{Schottky Diode}

The choice of power semiconductor device is very much useful in the power conversion circuit [13]. Since the rectifier of the rectenna system has to operate at microwave frequency, the power conversion device must have low reverse recovery time and the conversion efficiency must also be high. Schottky diode is chosen as a conversion device for the analysis of rectenna system in this paper.

Schottky diode named after the Genram scientist Walter G Schottky has low forward voltage drop and fast switching action. The cat's-whisker detectors used in the early days of wireless can be considered primitive Schottky diodes The barrier at the junction for a schottky diode is less when compared to that of $\mathrm{p}-\mathrm{n}$ junction device in both forward and reverse region[2]. This construction leads in high current at the same applied bias in the forward and reverse regions. Minority carriers are absent in Schottky diode and this leads to fast recovery and forms the basis for applications at high frequencies [1]. The above property of schottky diode leads to various applications for rectification purpose in rectenna system, for example in satellite solar power station.

The voltage drop across the diode terminals in case of Schottky is very less ( 0.6 to 1.7 volts) whereas the p-n junction diode has more. This lower voltage drop can provide higher switching speed and better system efficiency. Schottky diodes constructed from silicon carbide have a much lower reverse leakage current as compared to silicon Schottky diodes [4].

\section{TRANSMISSION LINES}

A transmission line is pair of conductors used in communication systems to carry high frequency signals and primarily used in communication systems to connect the system to the antenna.

Generally, its construction depends upon the parameters such as conductor dimensions, spacing and impedance matching to carry electromagnetic signals with minimal reflections and power losses [1]-[5]. Types of transmission line are parallel wire, coaxial cable, dielectric slabs, strip line, optical fiber, and waveguides. The frequency of operation determines which transmission line to be used. For millimeter waves, the transmission line used is optical cable. In this 
research the transmission line used is micro strip and co planar waveguides.

\section{Micro Strip Transmission Line (MLIN)}

Micro strip transmission lines are made up of conductors of thin strips on a sheet if dielectric substrate on the ground plate [1]. The open nature of the transmission line leads to radiation loss along with the dielectric loss in the substrate and the Ohmic loss in the strip conductor and the ground plane due to finite conductivity [12]. In this paper a micro strip transmission system has relative dielectric constant $\left(\varepsilon_{\mathrm{r}}\right)$ equal to 4.5 is on one side while a $\varepsilon_{\mathrm{r}}$ of 1 (for air) is on the other side. In a micro strip circuit the conductor is parallel to the ground plane.

It can be made by having a strip of copper on one side of a printed circuit board (PCB) or ceramic substrate while the other side is a continuous ground plane. The characteristic impedance depends in the physical and electrical characteristics of the micro strip line. The physical characteristics include the thickness of the insulating layer (PCB or ceramic) and the electrical characteristics include dielectric constant. The micro strip transmission line is open whereas the coaxial cable is closed structure.

\section{Co-Planar Waveguide (CPW)}

In a coplanar waveguide, center strip can be formed by conductors which get separated by the two ground planes. The characteristic impedance, attenuation of the line and the dielectric constant can be determined from the dimensions of center strip, gap, thickness and permittivity of the substrate. The dielectric substrate thickness is optimized for low radiation. The CPW has good properties like zero cutoff, Quasi TEM lower order propagation mode along with operations at $50 \mathrm{GHz}, 100 \mathrm{GHz}$ range which makes it suitable for many applications including MMIC [6]. Other applications using CPW structures are micro-electromechanical systems (MEMS) based switches, phase shifters, filters, printed circuit antenna sensor and characterization jigs for material measurements [11].

\section{CPW Advantages}

The advantages can be numbered as given below [15]

1. Low power inductance

2. Structural strength by bottom Immediate

3. Shorter delivery path

4. Lower conductor loss and dispersion

5. Good isolation

6. Smaller, cheaper, simpler manufacturing procedure

\section{APPLICATIONS}

The applications of rectenna are many in number. The inventor of the rectenna used it to power a small helicopter and various energy organizations are using it harvest the solar energy indirectly. Other applications include Radio Frequency Identification (RFID) contact less smart cards, etc.

\section{Simulation Results AND Discussion}

The full-wave rectenna without low pass filter meets the man aired vehicles requirement because of its higher conversion efficiency and constant output power [9].

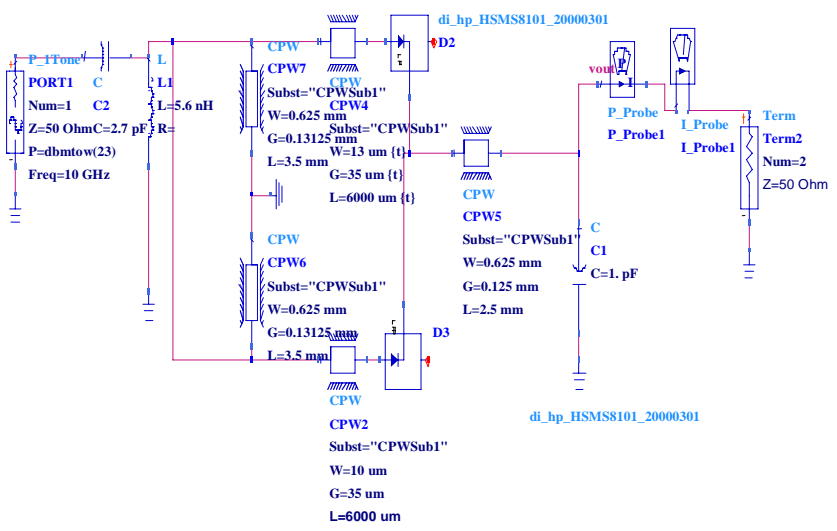

Figure 3: Design of Rectenna using CPW by ADS

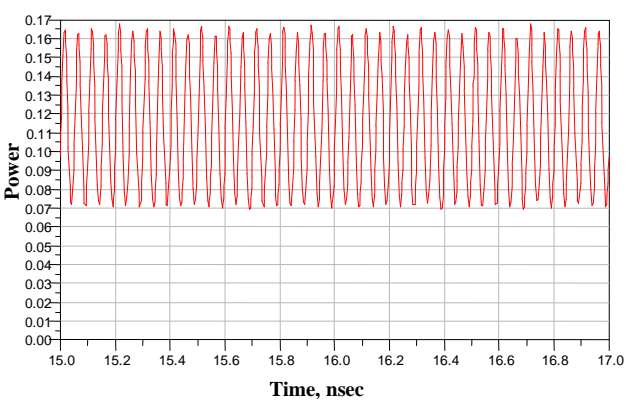

Figure 4: Simulation Result for Power Vs Time using CPW

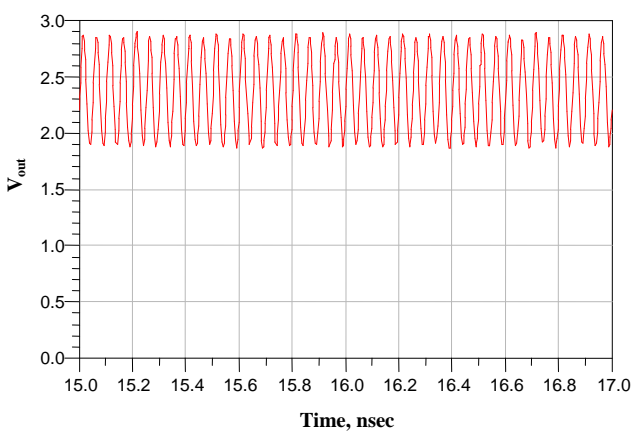

Figure 5: Simulation Result for Vout Vs Time using CPW 


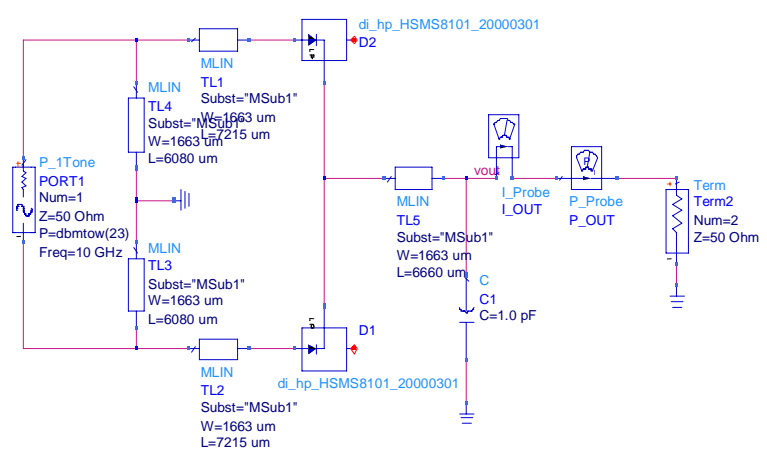

Figure 6: Design of Rectenna using MLIN by ADS

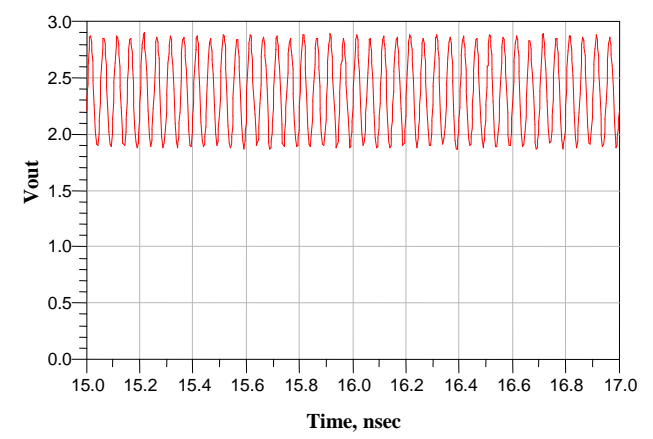

Figure 7: Simulation Result for Vout Vs Time Using MLIN

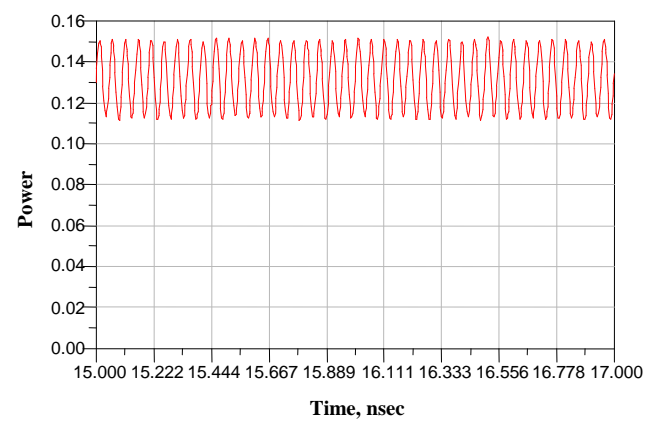

Figure 8: Simulation Result for Power vs Time Using MLIN

Fig 3 shows the schematic diagram of rectenna using CPW with the help of probe components, schottky diode, and coplanar waveguide as a transmission line and power source. Whereas Fig 4 shows output power of the rectenna of about $157 \mathrm{~mW}$ for an input power of $23 \mathrm{dBm}$ whereas Fig 5 shows the voltage oscillates from $2.2 \mathrm{~V}$ to $2.8 \mathrm{~V}$. Fig 6 shows the schematic diagram of rectenna using MLIN [16] with the help of micro strip line as a transmission line, probe component, schottky diode and power source. Fig 7 shows the output power of the rectenna of about $131 \mathrm{~mW}$ for an input power of $23 \mathrm{dBm}$ whereas Fig 8 shows the voltage oscillates from 2.3 to $2.75 \mathrm{~V}$. From this analysis, we came to know that the CPW based rectenna performs well when compared with MLIN based rectenna.

\section{CONCLUSION}

In this research a rectenna is designed and simulated with micro strip transmission line and coplanar waveguide as feeders. The simulated system shows improvement in the conversion efficiency and also our system provides less interference, low complexity and low cost. We also did the comparison of rectifying antenna using different transmission lines such as MLIN and CPW which produce good result compared with the existing rectenna system. Thus, we conclude that rectifying antenna with CPW has high conversion efficiency of about $78.5 \%$ when compared with rectenna using MLIN which has conversion efficiency of $65.5 \%$. Thus the improved efficiency is of about $13 \%$ which leads to application in wireless power transmission. The rectenna simulation results are done in Advanced Design System software 2009 from Agilent Technologies. In future, the efficiency can be improved when matching circuits and array of antennas can be implemented in the rectenna circuit design.

\section{REFERENCES}

[1] D.M. Pozar, Microwave Engineering, Third Edition, John Wiley \& Sons: New York, 2005

[2] L. H. Toh, "A Follow-up Study on Wireless Power Transmission for Unmanned Air Vehicles," Naval Postgraduate School, Master's Thesis, December 2007.

[3] http://en.wikipedia.org/wiki/Rectenna

[4] W. C. Brown, "The History of Wireless Power Transmission ", vol. 56, Pp. 3-21, 1996.

[5] Annapurna Das, Sisir K Das, "Microwave Engineering", Tata Mc Graw Hill, 2000.

[6] Canan Kavlak, Yasar Gurbuz and Ibrahim Tekin "A Coplanar Waveguide On-chip RF Choke for WLAN RF Circuits" Sabanci University, Faculty of Engineering and Natural Sciences, Orhanli, Tuzla 34956, Istanbul, Turkey

[7] Y. J. Ren and K. Chang, "5.8-GHz Circularly Polarized Dual-Diode Rectenna and Rectenna Array for Microwave Power Transmission", IEEE Trans. On Microwave Theory and Techniques, Vol. 54, No. 4, Pp.1495-1502, April 2006.

[8] S. Riviere, F. Alicalapa, A. Douyere, and J. D. Lan Sun Luk, "A compact rectenna device at low power level", Progress In Electromagnetics Research C, Vol. 16, Pp137-146, 2010.

[9] Agilent technologies, Advanced Design System, "Tutorial guide", 2009.

[10] César Meneses Ghiglino, "Ultra-Wideband (UWB) rectenna design for Electromagnetic Energy Harvesting", Master's thesis, Universitat Politècnica de Catalunya October, 2010.

[11] http://www.microwaves101.com/encyclopedia/coplanarwaveguide.cfm.

[12] http://en.wikipedia.org/wiki/Microstrip.

[13] http://en.wikipedia.org/wiki/Schottky_diode

[14] Rakesh Kumar Yadav, Sushrut Das, R. L. Yadava, "Rectenna Design Development and applications", International Journal of Engineering Science and Technology (IJEST), Vol. 3 No. 10 October 2011.

[15] Mohd Muhaiyiddin Bin Abdullah, "Analysis and design of CPW for High-speed pulse propagation on PCB", Master's thesis, University Sains Malaysia, 2007.

[16] Chun-Yi Liu," An improved design of rectenna for wireless power transmission for unmanned air vehicles" Naval Postgraduate School, Master's Thesis, September 2011 . 


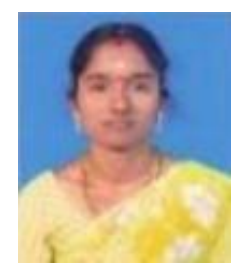

Mrs.S. Banu was born on $26^{\text {th }}$ January 1989 in Tamilnadu, India. She holds a bachelors degree in Electronics and Communication Engineering from Anna University, LCR college of Engineering and Technology,Tiruttani during 2010 and doing masters degree in Wireless Communication Systems in Periyar Maniammai University, Vallam, Thanjavur, TamilNadu. To her credit she has one publication in impact factor journal and four conference proceedings. Her areas of interest are microwave engineering and RF/Antenna design. Her current research is on RF/Antenna designing. She can be reached on rememberbanu@gmail.com

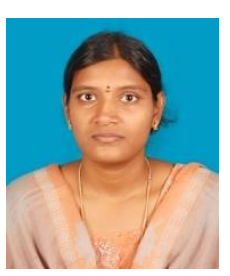

Ms.A. Vishwapriya was born on $22^{\text {nd }}$ June 1990 in Tamilnadu, India. She holds a bachelors degree in Electronics and Communication Engineering from Anna University-Coimbatore, PGP college of Engineering and Technology, Namakkal, Tamilnadu during 2011 and doing masters degree in Wireless Communication Systems in Periyar Maniammai University, Vallam, Thanjavur, Tamilnadu. To her credit she has one publication in impact factor journal and four conference proceedings. Her areas of interest are communication systems, microwave engineering. Her current research is on RF/Antenna designing. She can be reached on vishwaa.nivi@ gmail.com

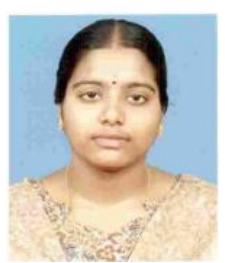

Ms.R. Yogamathi was born on $2^{\text {nd }}$ August 1990 in Tamilnadu, India. She holds a bachelors degree in Information Technology from Anna University-Trichy, Anjalai Ammal Mahalingam Engineering College during 2011 and doing masters degree in Wireless Communication Systems in Periyar Maniammai University, Thanjavur. To her credit she has one publication in impact factor journal and four conference proceedings. Her areas of interests are OFDM and RF/Antenna design. Her current research is on RF/Antenna designing. She can be reached on yoga.mathi@yahoo.com

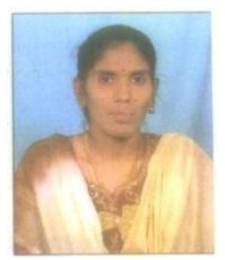

Mrs.A.V. Meenakshi was born on $7^{\text {th }}$ June 1977 in Tamilnadu, India. She holds a bachelors degree in Electronics and Communication Engineering from Madras University, Government College of Engineering during 1998, a master's degree in Communication Systems from Anna University, Thiagarajar College of Engineering, Madurai during 2004. She is currently working as an Assistant professor in Periyar Maniammai University, Thanjavur, India. She has more than 10 years of experience in teaching engineering in Tamilnadu, India. She has also authored or coauthored over 6 international journal papers and 13 international conference papers. Her current research interests and activities are in signal processing, RF components design and wireless communication. She can be reached on meenu_gow@yahoo.com

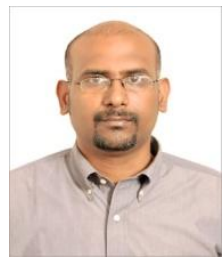

Dr.SPK. Babu was born on $2^{\text {nd }}$ December 1971 in Tamilnadu, India. He holds a bachelors degree in Electronics and Communication Engineering from Bharathiar University during 1995, a master's degree in Communication Systems from Madurai Kamaraj University during 1998 and $\mathrm{PhD}$ from University Sains Malaysia, Penang, Malaysia during 2010. He has more than 13 years of experience in teaching engineering in India and Malaysia. To his credit he has two publications in impact factor journal and few conference proceedings. He is a life member of Indian Society for Technical Education. His research interests include signal processing for digital communication, antenna design and soft computing for communication systems. Mail id: spkbabu@pmu.edu 